\title{
Inhibition of the endoplasmic reticulum (ER) stress-associated IRE-1/XBP-1 pathway alleviates acute lung injury via modulation of macrophage activation
}

\author{
Yanfeng Zhao ${ }^{1 \#, ~ Y a n ~ J i a n g ~}{ }^{1 \#}$, Linsong Chen ${ }^{1 \#}$, Xinlin Zheng ${ }^{2}$, Junjie Zhu ${ }^{1}$, Xiao Song ${ }^{1}$, Jinghan Shi ${ }^{1}$, \\ Yuping $\mathrm{Li}^{1}$, Wenxin $\mathrm{He}^{1}$ \\ ${ }^{1}$ Department of Thoracic Surgery, Shanghai Pulmonary Hospital affiliated to Tongji University, Shanghai 200433, China; ${ }^{2}$ Department of Thoracic \\ Surgery, Weifang Hospital of Traditional Chinese Medicine, Weifang 261041, China \\ Contributions: (I) Conception and design: Y Li, W He; (II) Administrative support: W He; (III) Provision of study materials or patients: Y Zhao, Y Li, \\ W He; (IV) Collection and assembly of data: Y Zhao, Y Jiang, L Chen, X Zheng, J Zhu, X Song, J Shi; (V) Data analysis and interpretation: L Chen, \\ X Zheng, J Zhu, X Song, J Shi, Y Li, W He; (VI) Manuscript writing: All authors; (VII) Final approval of manuscript: All authors. \\ \#These authors contributed equally to this work. \\ Correspondence to: Yuping Li; Wenxin He. Department of Thoracic Surgery, Shanghai Pulmonary Hospital affiliated to Tongji University, Shanghai \\ 200433, China. Email: liyuping2518@163.com; awen.he@yahoo.com.
}

Background: Both endoplasmic reticulum (ER) stress and macrophage diversity contribute to inflammatory processes in lung injury. However, the interaction between ER stress and macrophage M1/M2 imbalance in lung inflammation remains unclear. The present study, thus, aimed to evaluate the role of ER stress-mediated macrophage phenotype changes in lipopolysaccharide (LPS)-induced acute lung injury (ALI). Methods: Lung inflammation and injury were examined in a murine model of LPS-induced ALI with or without ER stress inhibitors. Alveolar macrophage (AM) polarization was determined by flow cytometry. Bone marrow-derived macrophages (BMDMs) were treated with either an ER stress inducer, inhibitor, or an IRE-1 endonuclease inhibitor before being polarized to an M1 and M2 phenotype. The macrophage polarization status was examined via RT-PCR and flow cytometry.

Results: Our results indicated that ER stress and IRE-1/XBP-1 signaling are activated in LPS-induced ALI. Furthermore, we observed that AM polarizes to an inflammatory phenotype upon exposure to LPS in the induction phase and an anti-inflammatory phenotype in the resolution phase of lung inflammation. Inhibition of ER stress attenuated the pathophysiological features of LPS-induced lung inflammation/injury, as evidenced by a decrease in bronchoalveolar lavage (BAL) protein levels, the number of inflammatory cells, and the expression level of inflammatory mediators. In addition, the ER stress inducer promoted M1 polarization and the switch from M2 to M1 in BMDMs, whereas inhibition of ER stress and XBP-1 splicing suppressed M1 but did not promote M2, both in vivo and in vitro.

Conclusions: Our results demonstrated that inhibition of the ER stress-associated IRE-1/XBP-1 signaling pathway suppresses M1 polarization and ameliorates LPS-induced lung injury. This indicates that the interaction between ER stress and macrophage polarization might be a novel therapeutic target for endotoxin-induced lung inflammatory disorders.

Keywords: Acute lung injury (ALI); endoplasmic reticulum stress (ER stress); inflammation; lipopolysaccharide (LPS); macrophage polarization

Submitted Nov 24, 2019. Accepted for publication Jan 07, 2020.

doi: $10.21037 /$ jtd.2020.01.45

View this article at: http://dx.doi.org/10.21037/jtd.2020.01.45

(C) Journal of Thoracic Disease. All rights reserved. 


\section{Introduction}

Acute lung injury (ALI) and its most severe form, acute respiratory distress syndrome (ARDS), is characterized by profound inflammation and tissue injury, often resulting from systemic infections, lung infections, or trauma $(1,2)$. The mortality rate of ALI/ARDS remains unacceptably high, with the average reported in-hospital mortality rate ranging from $30 \%$ to $50 \%$. In addition to increased mortality during and after hospital discharge, patients who survive ARDS are likely to experience significant long-term sequelae (3).

Macrophages are key contributors to both the induction and resolution of lung inflammation (4). Based on their phenotype and function, macrophages have been broadly classified into classically activated (M1) and alternatively activated (M2) macrophages. Macrophages are polarized to the M1 phenotype by interferon- $\gamma$ or lipopolysaccharide (LPS) and this phenotype is generally characterized by a high production of pro-inflammatory factors, such as interleukin $1 \beta$ (IL-1 $\beta$ ), IL-6, IL-12, tumor necrosis factor- $\alpha$ (TNF- $\alpha$ ), and inducible nitric oxide synthase (iNOS). M1 macrophages are important for the direct host-defense against pathogens $(5,6)$. The M2 phenotype is induced by exposure to IL-4, IL-13, IL-10, as well as glucocorticoids, and its hallmark is the production of anti-inflammatory molecules, including IL-10, found in the inflammatory zone (Fizz-1), chitinase-like 3 (Ym-1), and arginase-1 (Arg-1). In contrast to M1 macrophages, M2 macrophages coordinate a series of regulated responses to abrogate inflammation and enhance tissue repair (5-8).

A growing number of studies have shown that macrophage M1/M2 imbalance is implicated in the development and recovery of ALI. For example, neutrophil extracellular traps (NETs) promote ARDS inflammation during the acute phase by inducing macrophage polarization to the M1 phenotype (9). MCP-induced protein 1 (MCPIP1) plays a protective role in sepsis-induced ALI by modulating macrophage polarization to the $\mathrm{M} 2$ phenotype via inhibition of the JNK/c-Myc signaling pathway (10). Moreover, the promotion of the M2 phenotype was shown to have a protective role in endotoxemia and LPS-induced ALI $(11,12)$. These findings suggest that inhibition of M1 and/or promotion of M2 may have a critical therapeutic value in alleviating ALI.

Endoplasmic reticulum (ER) stress is defined as the accumulation of unfolded or misfolded proteins in the ER, which induces a coordinated adaptive process called the unfolded protein response (UPR). In mammalian cells, the
UPR pathway involves three primary signaling cascades initiated by the ER-localized transmembrane proteins: the protein kinase receptor-like ER kinase (PERK), prototranscription factor activating transcription factor 6 (ATF6), and inositol-requiring enzyme-1 (IRE-1). In response to ER stress, PERK activation regulates cellular protein synthesis via phosphorylation of the eukaryotic translation initiation factor $2 \alpha$ (eIF2 $\alpha$ ). IRE-1 triggers changes in downstream signaling by splicing 26 nucleotides from the XBP-1 mRNA, thereby causing a frameshift in its translation. ATF-6 leaves the ER and traffics to the Golgi, where it is processed to an active form. Activation of these signaling cascades leads to the upregulation of various UPR target genes to restore ER homeostasis (13).

In addition to its intrinsic effects on protein translation and folding, ER stress is linked to lung inflammatory diseases. A number of studies have shown that GRP78, an ER stress marker and UPR mediators are enhanced in both ALI/ARDS patients and rodent models of LPS-induced ALI (14-16). Moreover, inhibition of ER stress has been shown to alleviate endotoxin-induced ALI both in vivo and in vitro, thereby underlining the potential importance of this pathway in lung inflammation $(15,16)$. Thus, ER stress may be a central component of lung inflammatory diseases.

Therefore, both ER stress and M1/M2 imbalance contribute to inflammatory processes in lung injury. However, little is known regarding the interaction between ER stress and M1/M2 imbalance in lung inflammation. In this study, we evaluated the possible role of the M1/M2 imbalance in LPS-induced lung inflammation, focusing on the crosstalk between ER stress and macrophage phenotype change in LPS-induced ALI.

\section{Methods}

\section{Materials}

LPS (Escherichia coli 055:B5), tauroursodeoxycholic acid (TUDCA), and $4 \mu 8 \mathrm{c}$ were purchased from Sigma (St. Louis, MO, USA). Recombinant M-CSF and IL-4 were purchased from PeproTech (Rocky Hill, NJ, USA). All cell culture reagents were purchased from Invitrogen (Carlsbad, CA, USA). The protease and phosphatase inhibitor cocktails, BCA protein assay kit, enhanced ECL chemiluminescence reagent, and Western blot stripping buffer were purchased from Thermo Scientific (Rockford, IL, USA). Anti-GRP78 and anti-ATF6 antibodies were purchased from Abcam (Cambridge, MA, USA), while anti-XBP-1s, anti-eIF2 $\alpha$, and 
anti-p-eIF $2 \alpha$ antibodies were purchased from Cell Signaling Technology (Danvers, MA, USA). The $\beta$-actin-peroxidase $\mathrm{mAb}$ was purchased from Sigma-Aldrich. PE-labeled Arg1 was purchased from R\&D Systems (Minneapolis, MN, USA), and eFluor 660-labeled CD170 (Siglec F), FITClabeled CD11c, and PE-Cy7 labeled iNOS were purchased from Affymetrix eBioscience (San Diego, CA, USA).

\section{LPS-induced ALI}

Male C57BL/6 mice (8-10-week-old) were purchased from Bi Kai Experimental Animals (Shanghai, China) and bred in the Animal Care Facility of Tongji University affiliated with the Shanghai Pulmonary Hospital. LPS-induced ALI was performed as previously described (11). Briefly, following anesthetization with pentobarbital, $50 \mu \mathrm{g}$ of LPS in a volume of $25 \mu \mathrm{L}$ of saline was delivered to the mice intratracheally using a MicroSprayer syringe assembly (MSA-250-M, Penn Century, USA). ALI control mice (Veh) received the same amount of sterile saline. For TUDCA treatment, a dose of $5 \mathrm{mg} / \mathrm{kg}$ of TUDCA was administered intraperitoneally $1 \mathrm{~h}$ before LPS treatment. Vehicle control mice received the same amount of sterile saline. At specific time points following LPS administration, the bronchoalveolar lavage fluid (BALF) and lung tissue samples were obtained for Western blotting and quantitative RTPCR analysis. In separate experiments, nonlavaged lungs were collected for histological study.

\section{BALF collection}

Immediately after being sacrificed by administering an anesthetic overdose, $500 \mu \mathrm{L}$ of PBS were slowly infused in the murine lungs via tracheostomy and, then, withdrawn gently. This lavage was repeated 3 times and the obtained BALF samples were mixed. The fluid was, then, centrifuged and the cell-free supernatant was aliquoted and stored at $-80{ }^{\circ} \mathrm{C}$ until use. For the pellet, total cells were either counted on a hemocytometer following lysis of erythrocytes or purified by adhesion to tissue culture plastic to obtain alveolar macrophages (AMs) (17).

\section{Bone marrow-derived macrophage (BMDM) culture}

Bone marrow cells were collected from the femurs and tibias of C57BL/6 mice and treated with a red blood cell lysis buffer. The remaining cells were cultured in DMEM medium supplemented with $10 \%$ (vol/vol) fetal bovine serum, M-CSF (20 ng/mL), penicillin (100 U/mL), and streptomycin $(100 \mathrm{U} / \mathrm{mL})$. On day 6 or 7 , BMDMs were replated and then untreated (M0) macrophages were stimulated with LPS $(100 \mathrm{ng} / \mathrm{mL})$ for $18 \mathrm{~h}$ (M1), or with IL-4 (20 ng/mL) for $18 \mathrm{~h}(\mathrm{M} 2)$. To induce ER stress, BMDMs were pretreated with $2.5 \mu \mathrm{M}$ thapsigargin ( $\mathrm{Tg}$ ) for 1 h. To inhibit ER stress and XBP-1 splicing, BMDMs were pretreated either with $50 \mu \mathrm{g} / \mathrm{mL}$ TUDCA or $5 \mu \mathrm{M} 4 \mu 8 \mathrm{c}$ (18) for $1 \mathrm{~h}$. After stimulation, cells were harvested and stained for flow cytometry analysis or subjected to quantitative RTPCR analysis.

\section{Western blot analysis}

Cell and lung samples were homogenized in RIPA buffer containing protease and phosphatase inhibitor cocktails. The total protein content in the tissue lysates was quantified using the BCA protein assay kit. Equal amounts of protein samples were separated by SDS-PAGE. Proteins were, then, transferred to a PVDF membrane (Bio-Rad, Hercules, CA, USA). The membranes were incubated overnight at $4{ }^{\circ} \mathrm{C}$ with primary antibodies diluted in TBST (TBS containing $0.1 \%$ Tween-20) buffer containing 3\% non-fat milk, followed by incubation with corresponding secondary antibodies for $1 \mathrm{~h}$ at room temperature. Immunoreactive bands were detected using the enhanced ECL chemiluminescence reagent. The membrane was stripped using the Western blot stripping buffer and subsequently labeled with $\beta$-actin antibodies following the procedure described above. Immunoreactive bands were captured using the Amersham Imager 600 software (GE Healthcare).

\section{RNA isolation and quantitative RT-PCR}

Total RNA was isolated using the TRIzol reagent (Invitrogen, Carlsbad, CA, USA), following the manufacturer's instructions. Along with genomic DNA (gDNA) removal, $500 \mathrm{ng}$ of RNA was reverse transcribed using the TransScript All-in-One First-Strand cDNA Synthesis Kit (Transgenbiotech, Beijing, China). RTPCR (SYBR) was performed using reagents from Transgenbiotech (Beijing, China) on a QS6 Real-Time PCR System (Applied Biosystems). Relative gene expression levels were calculated using the comparative threshold cycle (Ct) method and normalized to $\beta$-actin. The primers used for amplification are listed in Table 1. 
Table 1 List of primers used for quantitative RT-PCR

\begin{tabular}{|c|c|c|}
\hline Gene & Primer pair & Primer sequence \\
\hline \multirow[t]{2}{*}{$I L-1 \beta$} & Forward & 5'-GAAATGCCACCTITTGACAGTG-3' \\
\hline & Reverse & 5'-TGGATGCTCTCATCAGGACAG-3' \\
\hline \multirow[t]{2}{*}{ IL-6 } & Forward & 5'-CCAAGAGGTGAGTGCTTCCC-3' \\
\hline & Reverse & 5'-CTGTTGTTCAGACTCTCTCCCT-3' \\
\hline \multirow[t]{2}{*}{ IL-10 } & Forward & 5'-GCTCTTACTGACTGGCATGAG-3' \\
\hline & Reverse & 5'-CGCAGCTCTAGGAGCATGTG-3' \\
\hline \multirow[t]{2}{*}{$I L-12$} & Forward & 5'-TGGTTTGCCATCGTTTTGCTG-3' \\
\hline & Reverse & 5'-ACAGGTGAGGTTCACTGTTTCT-3' \\
\hline \multirow[t]{2}{*}{$T N F-\alpha$} & Forward & 5'-GACGTGGAACTGGCAGAAGAG-3' \\
\hline & Reverse & 5-TTGGTGGTTTGTGAGTGTGAG-3' \\
\hline \multirow[t]{2}{*}{$\operatorname{Arg}-1$} & Forward & 5'-CTCCAAGCCAAAGTCCTTAGAG-3' \\
\hline & Reverse & 5'-AGGAGCTGTCATTAGGGACATC-3' \\
\hline \multirow[t]{2}{*}{ Fizz-1 } & Forward & 5'-CCTGCTGGGATGACTGCTACT-3' \\
\hline & Reverse & 5'-AGATCCACAGGCAAAGCCAC-3' \\
\hline \multirow[t]{2}{*}{$Y m-1$} & Forward & 5'-CAGGTCTGGCAATTCTTCTGAA-3' \\
\hline & Reverse & 5'-GTCTTGCTCATGTGTGTAAGTGA-3' \\
\hline \multirow[t]{2}{*}{$\beta$-actin } & Forward & 5'-GGCTGTATTCCCCTCCATCG-3' \\
\hline & Reverse & 5'-CCAGTTGGTAACAATGCCATGT-3' \\
\hline
\end{tabular}

\section{Determination of cytokine levels}

IL-1 $\beta$, IL-6, IL-10, IL-12, MIP-1 $\alpha$, and TNF- $\alpha$ levels in the BALF samples were measured by Luminex 200 (BioRad, Hercules, CA, USA).

\section{Lung histological study}

Following treatment with TUDCA and LPS, mice were sacrificed by administering an anesthetic overdose. The left lungs were harvested and fixed with $4 \%$ paraformaldehyde. After tissue embedding and sectioning, lung slices were subsequently stained with hematoxylin and eosin (H\&E). The tissue sections were observed under a light microscope and images were captured using an Olympus IX73 microscope (Clarksburg, NJ, USA). To perform the histological assessment of lung injury, five independent variables were evaluated-interstitial edema, hemorrhage, neutrophil infiltration, atelectasis, and necrosis. The severity of injury was judged by an investigator blinded to the experimental protocol according to the following criteria: no injury $=0$; injury to $25 \%$ of the field $=1$; injury to $50 \%$ of the field $=2$; injury to $75 \%$ of the field $=3$ and diffuse injury $=4(19,20)$.

\section{Flow cytometry analysis}

Cells isolated from BALF samples were first incubated with Fc block (eBioscience) and, then, cell surface staining was carried out by incubation with APC-conjugated antimouse CD11c and FITC-conjugated anti-mouse Siglec F. Following fixation and permeabilization (BD Fixation and Permeabilization Solution Kit, BD Biosciences), intracellular staining was performed by incubation with PE-conjugated anti-mouse Arg-1 and PE-CY7-conjugated anti-mouse iNOS. The proper isotype controls were used in each case. Flow cytometry events were gated first based on forward and side scatter. Then, CD11 $\mathrm{c}^{+}$Siglec $\mathrm{F}^{+}$cells $(21,22)$ (for in vivo AMs) were selected to evaluate the expression of Arg-1 and iNOS, respectively. Each flow cytometry analysis experiment was run on at least 100,000 cells and data were acquired using a BD FACSCanto ${ }^{\mathrm{TM}}$ II instrument and analyzed using the FlowJo X software.

\section{Statistical analysis}

All data, unless otherwise indicated, are presented as the mean \pm standard deviation $(\mathrm{SD})$ of independent experiments. The statistical significance of the differences between the two groups was analyzed using Student's $t$-test. A P value of $\leq 0.05$ was considered to be statistically significant. All statistical analyses were performed using the GraphPad Prism 8 software program for Windows.

\section{Results}

\section{ER stress and the UPR signaling patbway were activated in LPS-induced ALI}

To investigate whether ER stress is involved in LPS-induced lung injury, we measured the protein levels of GRP78 and various UPR markers in a murine model of LPS-induced ALI. Within hours of tracheal instillation of LPS, mice developed severe lung injury characterized by increased protein concentrations and number of inflammatory cells in BALF samples when compared with those of vehicle mice (Figure 1A,B,C). Macrophage and neutrophil infiltration increased within $6 \mathrm{~h}$ after LPS administration and reached peak levels at $24 \mathrm{~h}$. A full recovery was achieved at day 11 

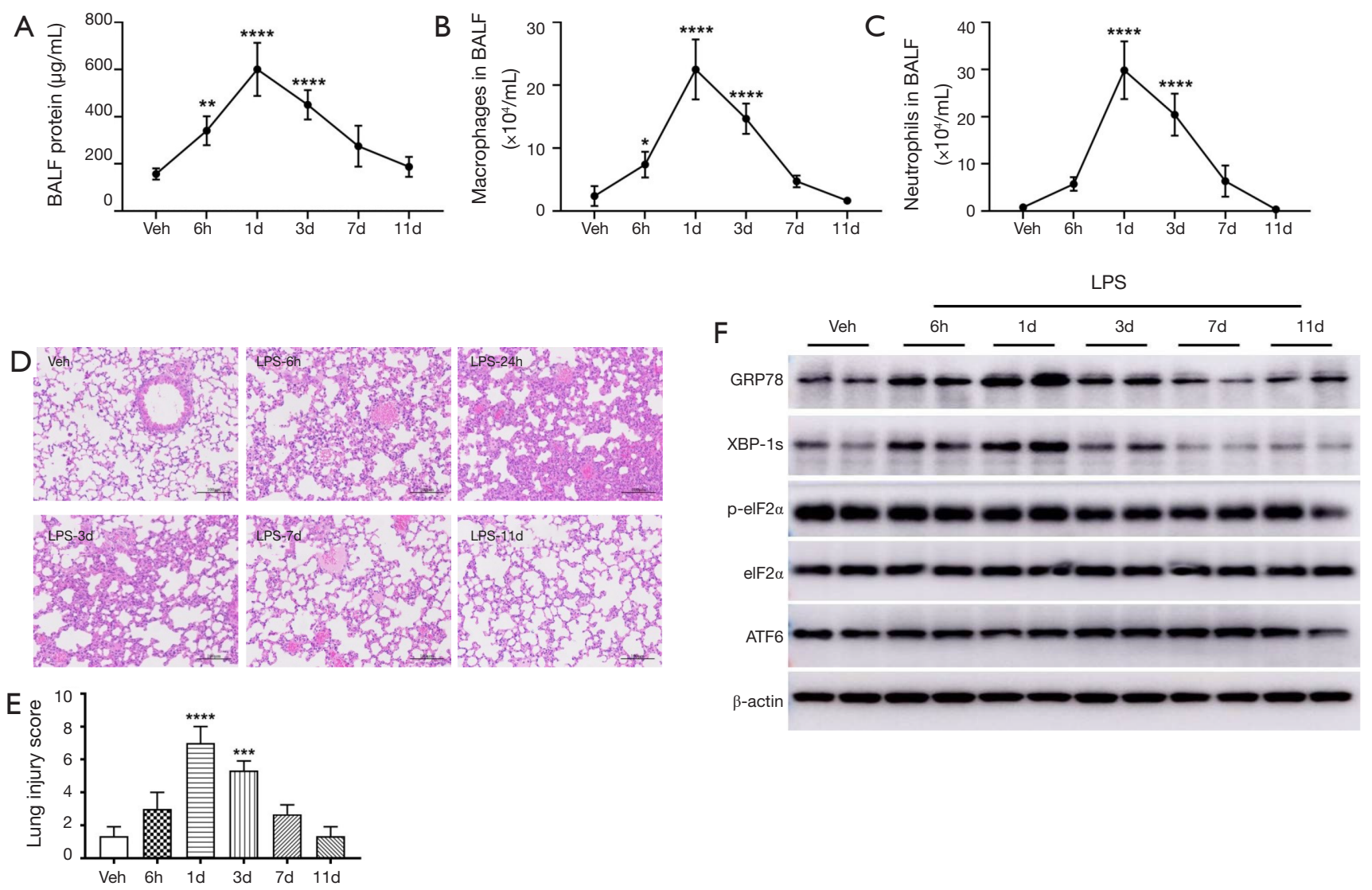

Figure 1 Endoplasmic reticulum (ER) stress and IRE-1/XBP-1 signaling were activated in LPS-induced acute lung injury (ALI). LPS was intratracheally administered in C57BL/6 mice at different time points. The protein concentration (A) and numbers of macrophages (B) and neutrophils (C) were measured in BALF samples. Representative H\&E stained sections (D) were obtained at different time points after LPS administration (magnification, $\times 200$ ). (E) The severity of lung injury was scored as described in Methods. (F) Protein levels of GRP78 and unfolded protein response UPR mediators were measured in lung tissues. $\beta$-actin was used as a control. Data are expressed as mean \pm SD for $\mathrm{n}=6-8$ mice. *, $\mathrm{P}<0.05 ;{ }^{* *}, \mathrm{P}<0.01$; ${ }^{* * *}, \mathrm{P}<0.001$; ${ }^{* * *}, \mathrm{P}<0.0001$ compared to the Veh group. LPS, lipopolysaccharide; UPR, unfolded protein response; BALF, bronchoalveolar lavage fluid; $\mathrm{SD}$, standard deviation.

(Figure $1 B, C$ ). Protein concentrations in the BALF also peaked at $24 \mathrm{~h}$ and significantly decreased on day 7 and 11 (Figure 1A). Consistently, histological analysis showed that the severity of lung injury, characterized by thickening of the alveolar septum, inflammatory cell infiltration, and alveolar fusion, peaked at $24 \mathrm{~h}$ after LPS administration and declined thereafter (Figure 1D,E).

We subsequently analyzed changes in ER stress mediators during the induction and resolution phases of ALI. As shown in Figure 1F, the protein expression of GRP78 was increased by LPS administration at $6 \mathrm{~h}$, peaked at $24 \mathrm{~h}$ and returned to the normal level on day 7 and 11. To further investigate which UPR components are involved in LPS-induced ER stress, three key proteins, ATF6, XBP-1, and phosphorylated eIF2 $\alpha$ (p-eIF2 $\alpha$ ), were detected by Western blotting. We found that the expression of p-eIF $2 \alpha$ and ATF6 proteins did not change significantly after LPS treatment, whereas LPS treatment induced the accumulation of spliced XBP-1 (XBP-1s) in lung tissues. This suggests that ER stress and IRE-1/XBP-1 signaling play an important role in LPS-induced ALI.

\section{Macrophage diversity was detected in the induction and resolution phase of $A L I$}

Considering that macrophage polarization is closely 
associated with lung inflammation and that M1-polarized macrophages exhibit a pro-inflammatory phenotype, we identified the phenotype of infiltrated macrophages in the LPS-induced ALI model. The concentration of inflammatory mediators in the BALF peaked at either 6 or $24 \mathrm{~h}$ following LPS administration and returned to basal levels on day 11 (Figure $2 A$ ). We also examined the gene expression levels of M1 and M2 markers in RNA isolated from AMs. As expected, the gene expression level of M1 markers (IL-1 $\beta$, IL-6, IL-12, and TNF- $\alpha$ ) peaked early at $6 \mathrm{~h}$ during the acute phase of inflammation and injury (Figure 2B). Unlike the M1 markers, the trends seen in the expression of M2 markers were different. Fizz1 appeared to have a bimodal distribution (Figure $2 B$ ) and peaked during both the induction and resolution phases of lung inflammation. Arg-1 expression peaked on day 3, while Ym-1 mRNA was significantly suppressed at 6 and $24 \mathrm{~h}$ after LPS administration and gradually returned to basal levels on day 11. Our results indicate that the AM population polarizes to an inflammatory M1 phenotype in the acute phase of inflammation and gradually shifts to an M2 phenotype in the resolution phase. Further, these results suggest that $M 1 / M 2$ imbalance plays an important role in the development and resolution of ALI.

\section{ER stress inbibitor TUDCA attenuated LPS-induced ALI by mitigating M1 macrophage polarization}

We next investigated the inhibitory effects of TUDCA (23), a potent ER stress inhibitor, on LPS-induced lung inflammation. As shown in Figure 3A, TUDCA treatment inhibited the protein expression of GRP78 and XBP-1s in LPS-instilled mice. Histological analysis revealed that LPS caused a significant increase in the inflammatory infiltrating cells and structural damage when compared with the vehicle control. However, TUDCA treatment attenuated these histological changes (Figure 3B). We also found that TUDCA treatment prevented lung vascular permeability in ALI, as assessed by the protein concentration in BALF samples (Figure 3C). The same trends were observed for the number of inflammatory cells in BAFL samples (Figure 3D) and inflammatory mediator levels in lung tissues (Figure $3 E$ ). At $24 \mathrm{~h}$ following LPS administration, the total number of cells in BALF samples and mRNA levels of IL$1 \beta$, IL-6, IL-10, and TNF- $\alpha$ were significantly increased. Following TUDCA administration, IL-10 levels slightly increased, while the total number of cells and IL-1 $\beta$, IL6 , and TNF- $\alpha$ levels decreased remarkably, indicating attenuated inflammation (Figure 3D,E). Consistent with these observations, flow cytometry analysis revealed a decreased expression of the M1 activation marker iNOS in the TUDCA treatment group when compared to the LPS group (Figure 3F). Moreover, TUDCA treatment did not promote the expression of the M2 activation marker Arg-1. There was no statistical difference between the vehicle and TUDCA administration alone groups.

\section{ER stress promoted M1 polarization and the switch from the M2 phenotype to the M1 phenotype in BMDMs}

To investigate how ER stress affects macrophage polarization, we treated BMDMs with the ER stressinducing drug, $\mathrm{Tg}$, followed by polarization to the M1 and M2 phenotypes. We found that induction of ER stress caused a further increase in the expression of proinflammatory M1 markers (IL-1 $\beta$, IL-6, and TNF- $\alpha$ ) in response to LPS stimulation. However, the gene expression of M2 markers (Fizz-1, and Ym-1) was inhibited in the presence of Tg (Figure 4A). Similarly, flow cytometry analysis revealed that $\mathrm{Tg}$ significantly enhanced iNOS expression in LPS-stimulated BMDMs, as shown by the right shift of the curve in the presence of $\mathrm{Tg}$, but inhibited Arg-1 expression in IL-4-stimulated BMDMs (Figure 4B).

\section{Blockade of ER stress and the XBP-1 signaling patbway inhibited M1 polarization in BMDMs}

To further confirm that ER stress promotes M1 polarization, we investigated the effects of an ER stress inhibitor on macrophage phenotype. Furthermore, we also evaluated whether the XBP-1 signaling pathway is involved in the macrophage phenotype shift. As expected, treatment with TUDCA and $4 \mu 8 \mathrm{c}$, which block substrate access to the active site of IRE- $1 \alpha$ and selectively inactivates XBP-1 splicing (18), respectively, inhibited LPS-induced M1 marker expression (IL-1 $\beta$, IL-6, and TNF- $\alpha$ ) (Figure $5 A$ ), but did not further enhance IL-4induced M2 marker gene expression (Fizz-1, and Ym1) in BMDMs (data not shown here). Consistent with these observations, flow cytometry analysis showed that treatment with both TUDCA and $4 \mu 8 \mathrm{c}$ significantly decreased the gene expression of iNOS in LPS-stimulated BMDMs, but did not enhance the gene expression of Arg-1 in IL-4-stimulated BMDMs (Figure 5B). There was no statistically significant difference between the M0, TUDCA, and $4 \mu 8 \mathrm{c}$ treatment alone groups 


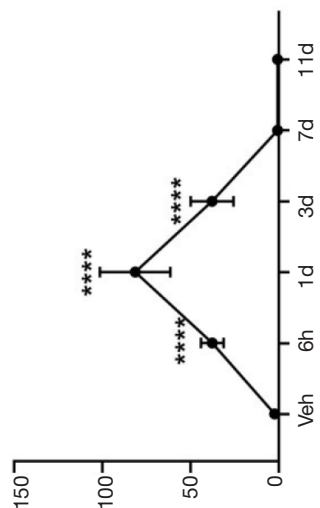

(ךu/6d)

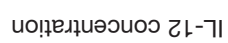

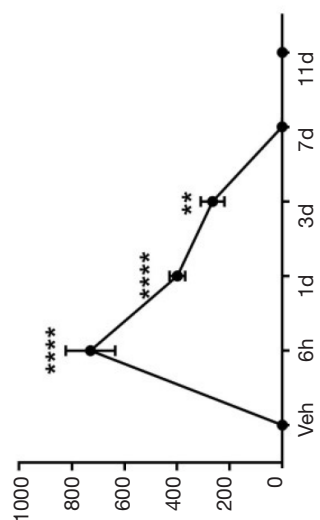

(ךw/6d)

иo!̣exұนəวиоо 9-기

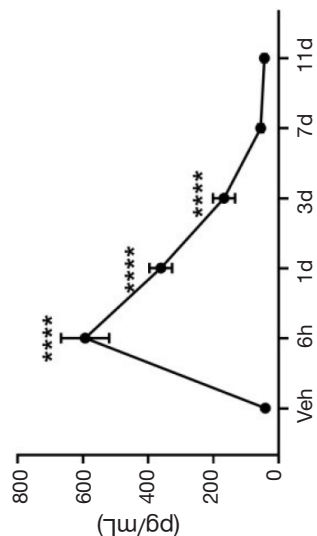

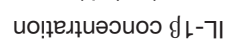
$\varangle$

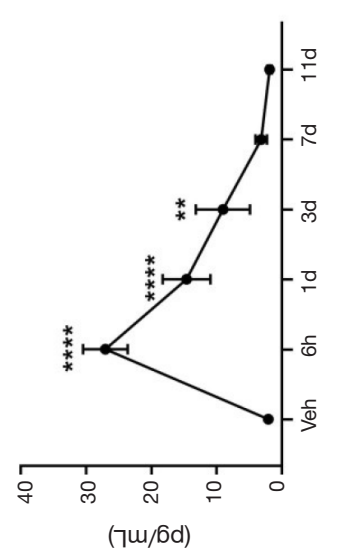

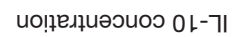

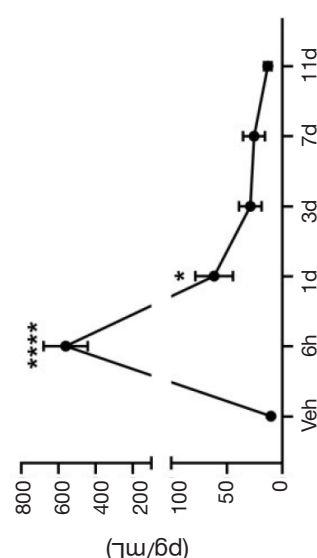

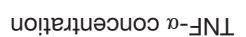

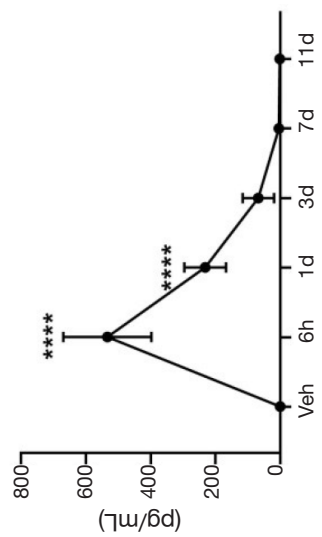

uo!̣eגұนəวนоว nL-dIW

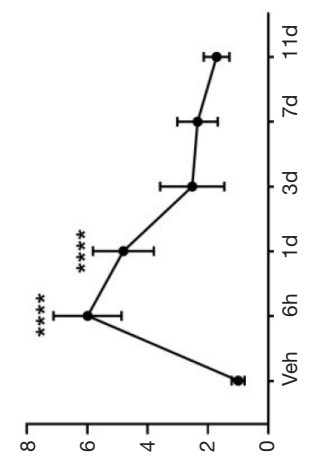

(чә^ 아 әм!ฺ리)

|әлә| $\forall N y$ س $n-\exists N \perp$

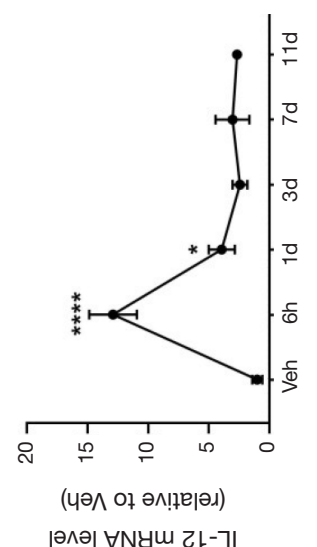

|әлә| $\forall N$ บั山 乙L-ר|

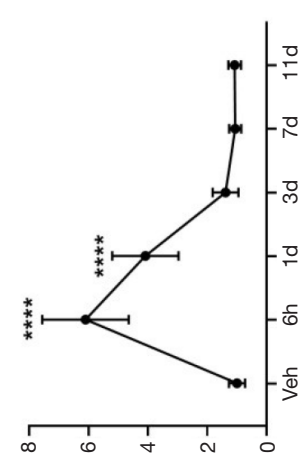

(4ә^ 아 әл!ฺв리)

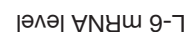
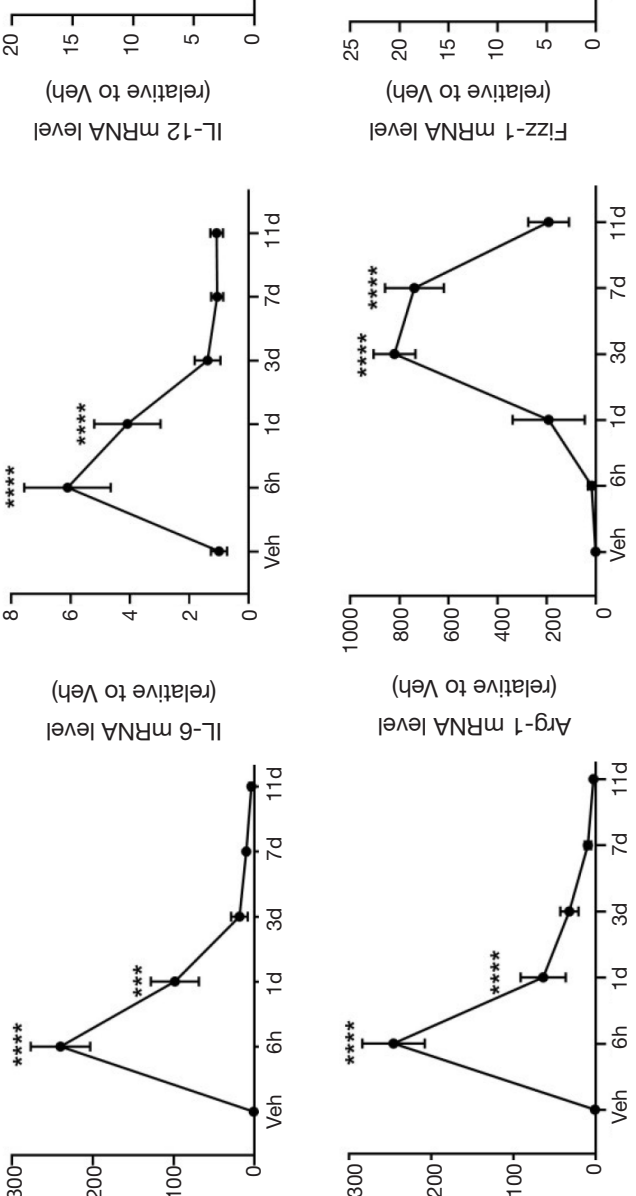

|әлә $\forall N y$ س [-ZZ!」

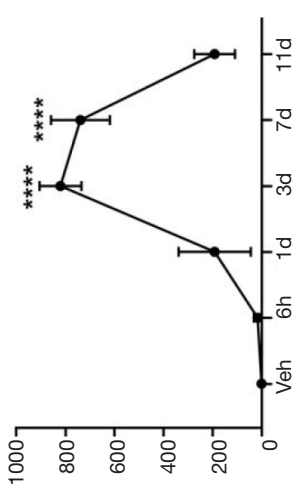

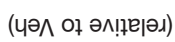

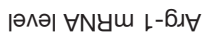

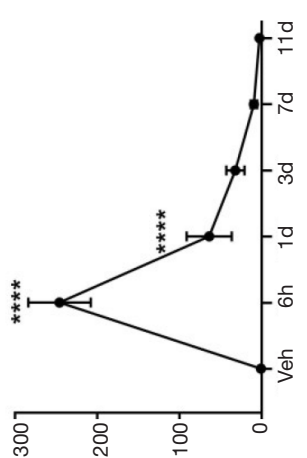

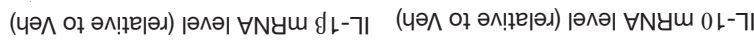

苞范

$\stackrel{8}{\circ}$

:

응

రㅇㅇㅇ

o

3 。

$\exists$ 离

㟧 不

当

E.

可

य

\pm ०

节

告

क ธี $v$

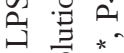

ت.

पँ

总

를

.

苗

踏

- I

岕至 $\frac{1}{8}$

\% $\Xi \frac{\pi}{5}$

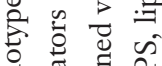

苞氙当 
A
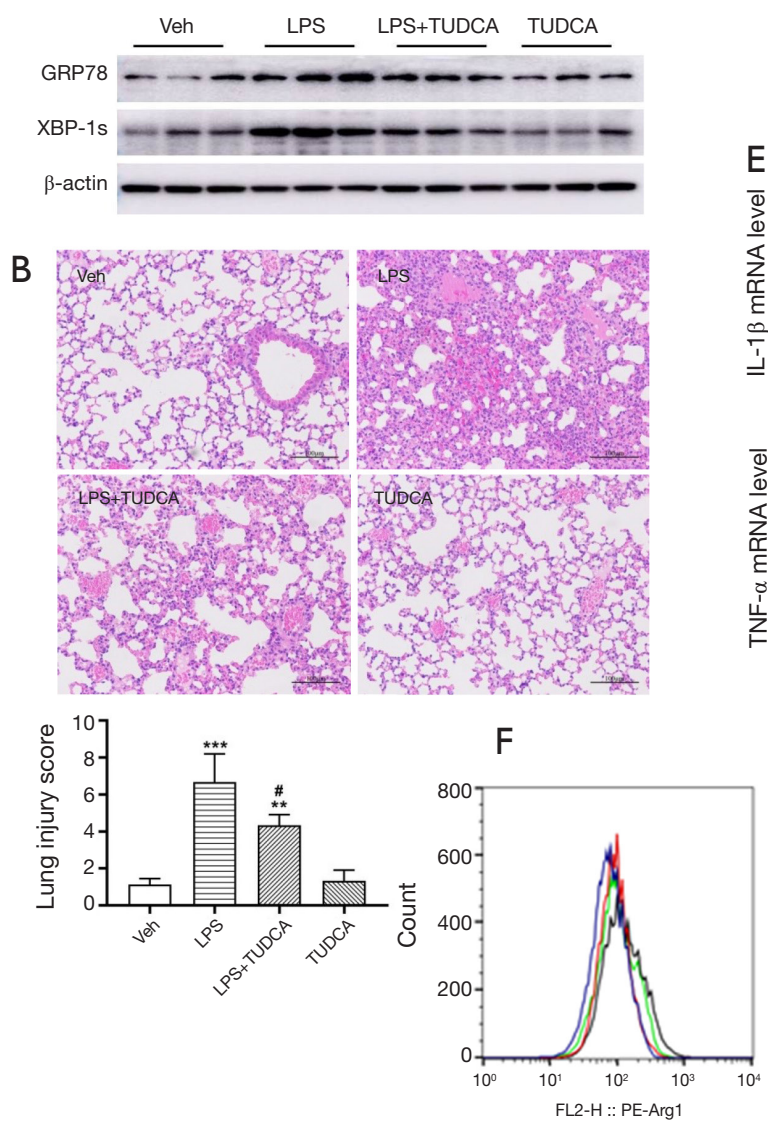

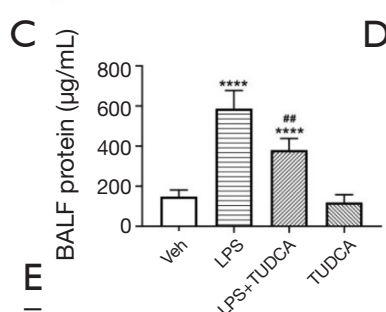

D

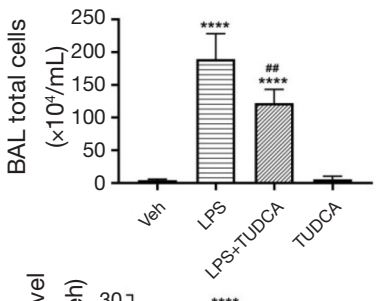

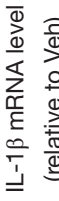
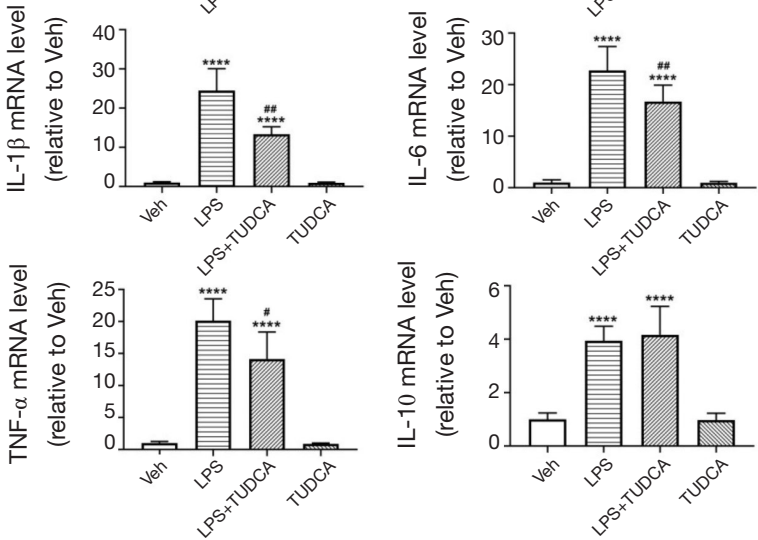
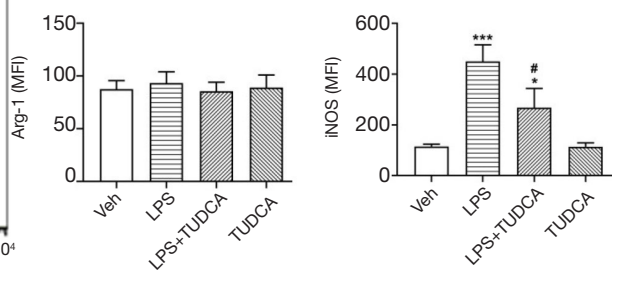

Figure 3 TUDCA mitigated M1 macrophage polarization levels in LPS-induced ALI. C57BL/6 mice subjected to LPS were treated with TUDCA for $24 \mathrm{~h}$. The protein levels of GRP78 and XBP-1s (A) were analyzed by Western blotting in lung tissues. H\&E staining (B) was performed in the lung sections (magnification, $\times 200$ ). The protein concentration (C) and the number of inflammatory cells (D) were measured in BALF samples. The gene expression of inflammatory mediators (E) was determined via RT-PCR. The protein levels of alveolar macrophage polarization markers (F) were determined by flow cytometry. Data are expressed as mean \pm SD for $n=6-8$ mice. *, $\mathrm{P}<0.05 ;{ }^{* *}, \mathrm{P}<0.01$; ***, $\mathrm{P}<0.001$; ****, $\mathrm{P}<0.0001$ compared to the Veh group; ${ }^{*}, \mathrm{P}<0.05 ;{ }^{* \#}, \mathrm{P}<0.01$ compared to the LPS group. TUDCA, tauroursodeoxycholic acid; LPS, lipopolysaccharide; ALI, acute lung injury; BALF, bronchoalveolar lavage fluid; SD, standard deviation.

(Figure 5A).

\section{Discussion}

ALI/ARDS, caused by either direct or indirect lung insults, can result in uncontrolled lung inflammation and structural changes that are associated with high morbidity and mortality $(1,2)$. Macrophage diversity contributes to both the promotion and resolution of lung inflammation. ER stress is a potent evolutionarily conserved response to misfolded proteins resulting from cellular stresses, such as inflammation. In this study, we demonstrated for the first time that the ER stress-mediated M1/M2 imbalance plays a role in promoting inflammation in ALI. Furthermore, we showed that inhibition of the ER stress-associated IRE-1/XBP-1 pathway suppresses M1 polarization, thus alleviating ALI.

ER stress has been implicated in a variety of diseases, including diabetes, atherosclerosis, neurodegenerative diseases, cancer, and inflammatory diseases $(24,25)$. In 

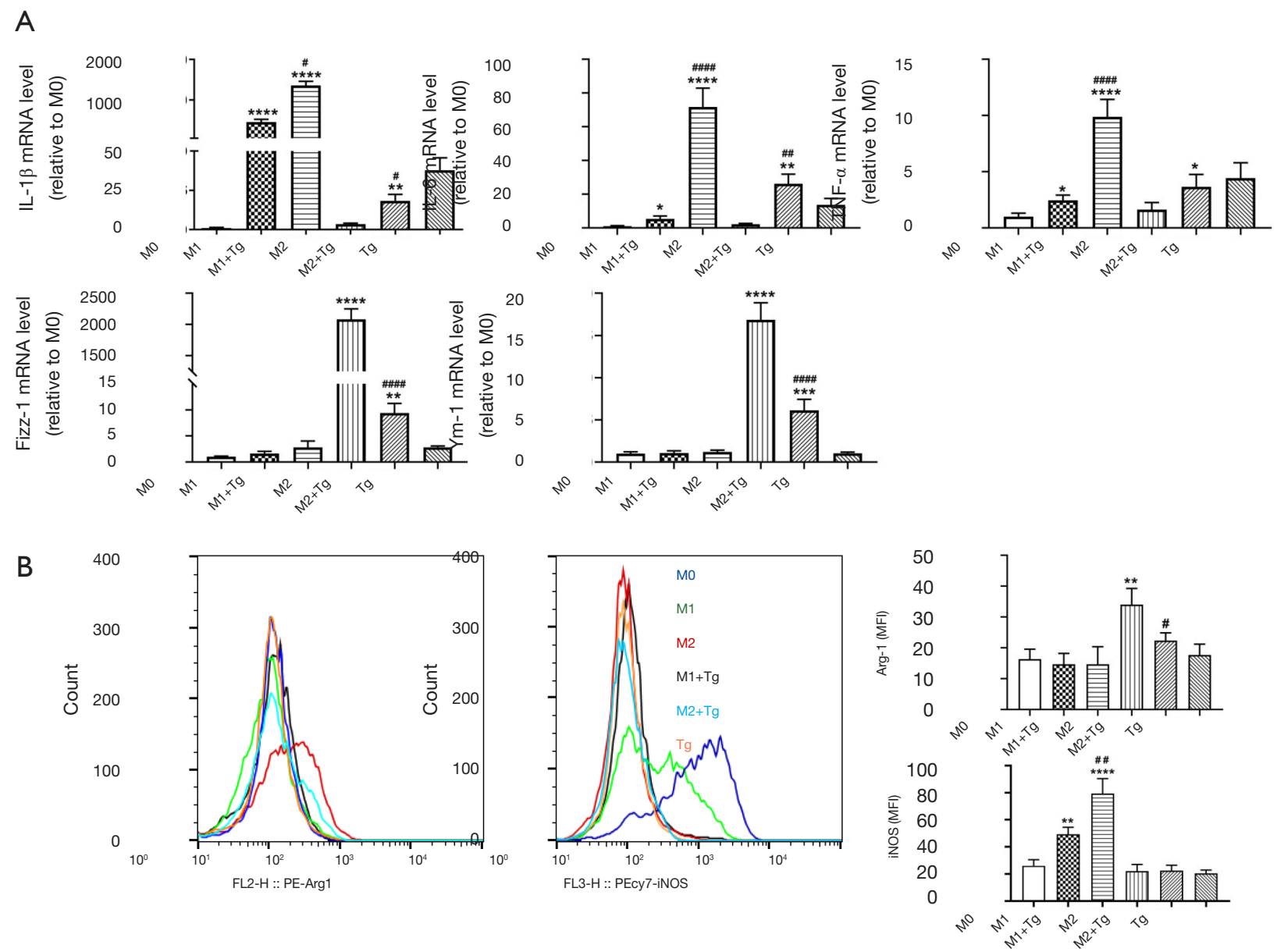

Figure 4 ER stress promoted M1 polarization in bone-marrow derived macrophages (BMDMs). Before being polarized to the M1 and M2 phenotypes, BMDMs were pretreated with Tg for $1 \mathrm{~h}$ as described in Methods. The gene expression of macrophage M1 and M2 markers (A) were determined by RT-PCR. The protein levels of macrophage polarization markers (B) were analyzed by flow cytometry. Data are

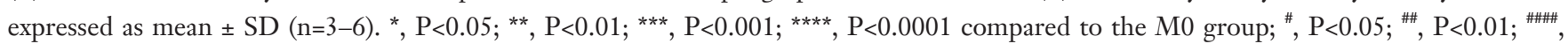
$\mathrm{P}<0.0001$ compared to either the M1 or M2 group. ER, endoplasmic reticulum; Tg, thapsigargin; SD, standard deviation.

particular, several studies have suggested a potential role of ER stress in the pathogenesis of lung injury. Saurabh and colleagues showed that patients with severe chronic obstructive pulmonary disease have elevated levels of the ER stress marker GRP78 (26). Enhancement of ER stress has been associated with the pathogenesis of idiopathic pulmonary fibrosis (27). In addition, ER stress is involved in LPS-induced lung injuries in vivo and is enhanced in LPSstimulated airway epithelial cells and thrombin-stimulated endothelial cells in vitro $(15,16,28)$. Our results also showed that the GRP78 protein levels were significantly increased following LPS administration at $6 \mathrm{~h}$ and peaked at $24 \mathrm{~h}$. Among the three UPR mediators, p-eIF2alpha, XBP-1, and ATF-6, only XBP-1s was significantly elevated in the lung tissues of LPS-treated mice. Furthermore, increased inflammatory cell infiltration and proinflammatory mediator secretion, as well as structural damage were observed in the lung of LPS-instilled mice. Moreover, we found that TUDCA, a potent ER stress inhibitor, markedly reduced the LPSinduced upregulation of GRP78 and XBP-1s in mice, as well as inhibited lung inflammation and histological changes. These findings suggest that the ER stress-associated XBP-1 pathway is one of the crucial players in the induction and maintenance of LPS-induced lung inflammation and that TUDCA attenuates ER stress in ALI.

Accumulating evidence has suggested that macrophage 

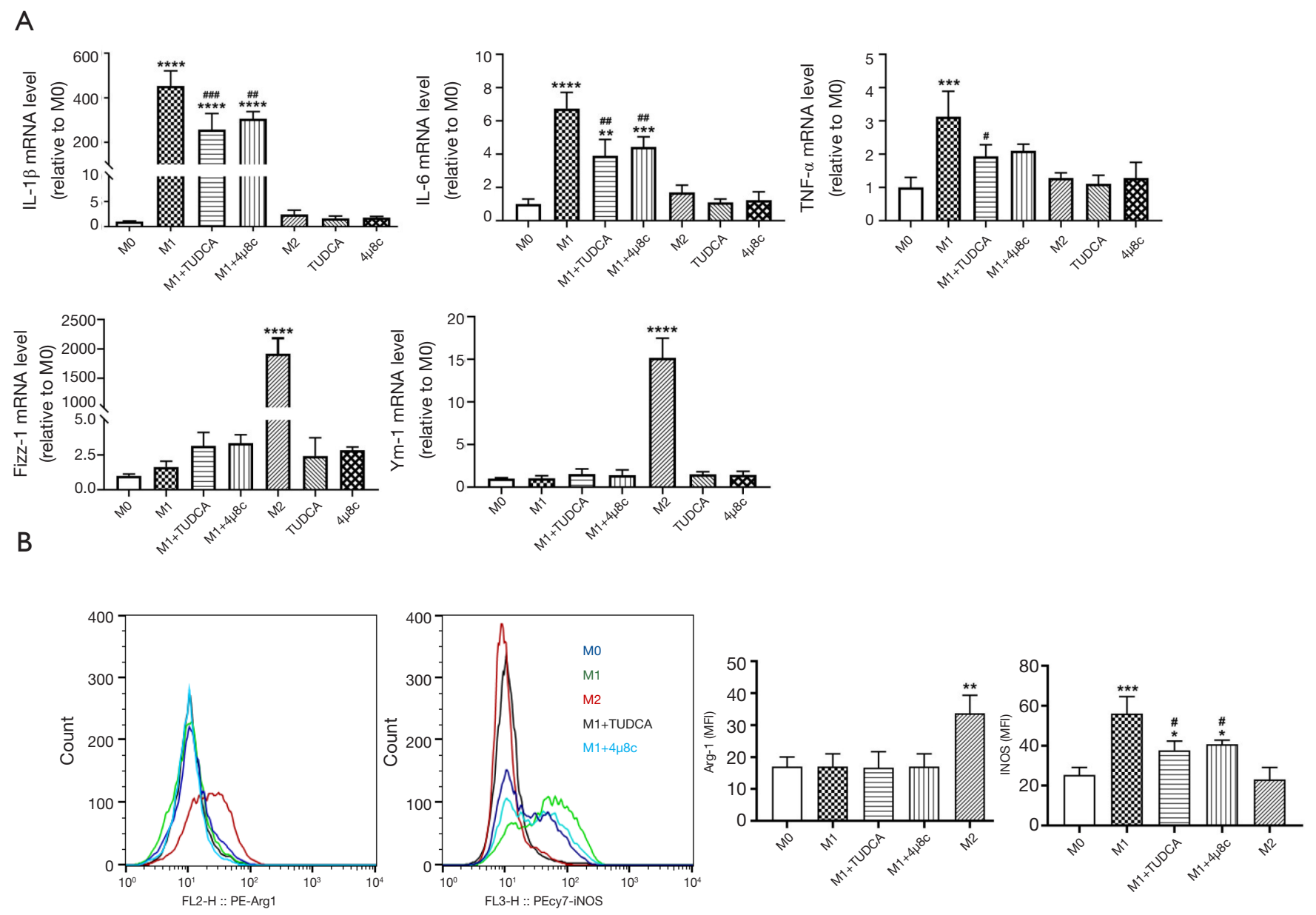

Figure 5 Inhibition of ER stress and XBP-1 signaling suppressed M1 polarization in BMDMs. Before being polarized to the M1 and M2 phenotype, BMDMs were pretreated with TUDCA and $4 \mu 8 \mathrm{c}$ for $1 \mathrm{~h}$ as described in Materials and Methods. The gene expression of macrophage M1 and M2 markers (A) was determined by RT-PCR. The protein levels of macrophage polarization markers (B) were analyzed using flow cytometry. Data are expressed as mean $\pm \mathrm{SD}(\mathrm{n}=3-6)$. ${ }^{*}, \mathrm{P}<0.05 ;{ }^{* *}, \mathrm{P}<0.01 ;{ }^{* * *}, \mathrm{P}<0.001 ;{ }^{* * * *}, \mathrm{P}<0.0001$ compared to the M0 group; ", $\mathrm{P}<0.05 ;$;\#, $\mathrm{P}<0.01$; ${ }^{\# \#}, \mathrm{P}<0.001$ compared to the $\mathrm{M} 1$ group. ER, endoplasmic reticulum; BMDMs, bone-marrow derived macrophages; TUDCA, tauroursodeoxycholic acid; MFI, mean fluorescence intensity; SD, standard deviation.

diversity is a key regulator of the development and recovery of ALI/ARDS and that different macrophage subtypes play different roles at different stages of ALI/ARDS $(4,22,29)$. Therefore, we analyzed the macrophage phenotype in a mouse model of LPS-induced lung injury. We found that the production of pro-inflammatory mediators (IL-1 $1 \beta$, IL6 , IL-12, MIP-1 $\alpha$, and TNF- $\alpha$ ) in BALF samples peaked at either 6 or $24 \mathrm{~h}$ following LPS administration. Interestingly, IL-10 levels were also elevated, possibly as a result of a large release of pro-inflammatory factors that activate antiinflammatory responses. Moreover, the mRNA of pro- inflammatory M1 markers (IL-1 $\beta$, IL-6, IL-12, and TNF- $\alpha$ ) peaked at $6 \mathrm{~h}$, during the acute phase of inflammation and injury. Unlike the M1 markers, the M2 gene panels (Fizz-1, Arg-1 and Ym-1) showed a different expression trend and were mostly enhanced in the resolution phase. Our data suggest that the AM population polarizes to an inflammatory macrophage phenotype upon exposure to LPS in the induction phase and to an anti-inflammatory macrophage phenotype in the resolution phase. Thus, restoring the M1/M2 imbalance via inhibition of the M1 phenotype and/or promotion of the M2 phenotype may 
have a critical therapeutic value in alleviating ALI.

Both ER stress and M1/M2 imbalance are potential therapeutic targets for various inflammatory diseases. Based on our current results, we speculate that ER stress may play a role in promoting M1 macrophage polarization. As expected, we found that $\mathrm{Tg}$ treatment caused either a further increase in pro-inflammatory M1 marker gene expression or a further decrease in anti-inflammatory M2 marker gene expression in BMDMs. In contrast, blockade of ER stress via TUDCA treatment generated the opposite effect in M1 signature markers but did not promote M2 signature markers. Consistent with the effect of TUDCA on macrophage polarization, the IRE-1 endonuclease inhibitor also demonstrated an inhibitory effect on M1 markers but did not promote the expression of M2 markers. These observations indicate that the blockade of the ER stress-associated IRE-1/XBP-1 signaling pathway alleviates ALI by inhibiting M1 polarization.

There are several limitations to our study. First, we could not address the cause-effect relationship between ER stress and inflammatory mediator secretion. Several studies indicate that not only does ER stress amplify inflammatory reactions but also that pro-inflammatory mediators, such as IL-1 $\beta$, IL-6, TNF- $\alpha$, and IL-17, contribute to the induction of ER stress (15,30-32). Our study demonstrated that inhibition of ER stress suppresses LPS-induced inflammation. Second, a proof-of-concept study is needed to determine the causal role of XBP-1 signaling in LPSinduced ALI using XBP-1 transgenic mice.

\section{Conclusions}

Taken together, our results demonstrate that inhibition of the ER stress-associated IRE-1/XBP-1 signaling pathway suppresses macrophage polarization to the M1 phenotype and ameliorates LPS-induced lung inflammation and injury. Moreover, our results suggest that the interaction between ER stress and macrophage phenotype might be a novel therapeutic target for endotoxin-induced lung inflammatory disorders.

\section{Acknowledgments}

Funding: This work was supported by the National Natural Science Foundation of China (No. 81400056 to Y Zhao, 81100061 to $W \mathrm{He}, 81670089$ to $\mathrm{W} \mathrm{He}$ and 81900078 to L Chen), Shanghai Municipal Commission of Health and Family Planning (No. 201540161 to Y Zhao, and 201640225 to $\mathrm{WHe}$ ).

\section{Footnote}

Conflicts of Interest: The authors have no conflicts of interest to declare.

Etbical statement: The authors are accountable for all aspects of the work in ensuring that questions related to the accuracy or integrity of any part of the work are appropriately investigated and resolved. All animal protocols were approved by the Animal Care and Use Committee of Shanghai Pulmonary Hospital (No. 2014fk12) and met the standards of the Institutional Health Guide for care and use of laboratory animals.

Open Access Statement: This is an Open Access article distributed in accordance with the Creative Commons Attribution-NonCommercial-NoDerivs 4.0 International License (CC BY-NC-ND 4.0), which permits the noncommercial replication and distribution of the article with the strict proviso that no changes or edits are made and the original work is properly cited (including links to both the formal publication through the relevant DOI and the license). See: https://creativecommons.org/licenses/by-nc-nd/4.0/.

\section{References}

1. Matthay MA, Ware LB, Zimmerman GA. The acute respiratory distress syndrome. J Clin Invest 2012;122:2731-40.

2. Han S, Mallampalli RK. The acute respiratory distress syndrome: from mechanism to translation. J Immunol 2015;194:855-60.

3. Shaw TD, McAuley DF, O'Kane CM. Emerging drugs for treating the acute respiratory distress syndrome. Expert Opin Emerg Drugs 2019;24:29-41.

4. Johnston LK, Rims CR, Gill SE, et al. Pulmonary macrophage subpopulations in the induction and resolution of acute lung injury. Am J Respir Cell Mol Biol 2012;47:417-26.

5. Martinez FO, Gordon S. The M1 and M2 paradigm of macrophage activation: time for reassessment. F1000Prime Rep 2014;6:13.

6. Murray PJ, Allen JE, Biswas SK, et al. Macrophage activation and polarization: nomenclature and experimental guidelines. Immunity 2014;41:14-20.

7. Wang J, Xie L, Wang S, et al. Azithromycin promotes alternatively activated macrophage phenotype in systematic lupus erythematosus via PI3K/Akt signaling pathway. Cell Death Dis 2018;9:1080. 
8. Shen Y, Song J, Wang Y, et al. M2 macrophages promote pulmonary endothelial cells regeneration in sepsis-induced acute lung injury. Ann Transl Med 2019;7:142.

9. Song C, Li H, Li Y, et al. NETs promote ALI/ARDS inflammation by regulating alveolar macrophage polarization. Exp Cell Res 2019;382:111486.

10. Zhang Y, Huang T, Jiang L, et al. MCP-induced protein 1 attenuates sepsis-induced acute lung injury by modulating macrophage polarization via the JNK/c-Myc pathway. Int Immunopharmacol 2019;75:105741.

11. Grailer JJ, Haggadone MD, Sarma JV, et al. Induction of M2 regulatory macrophages through the beta2-adrenergic receptor with protection during endotoxemia and acute lung injury. J Innate Immun 2014;6:607-18.

12. Chen X, Yang B, Tian J, et al. Dental Follicle Stem Cells Ameliorate Lipopolysaccharide-Induced Inflammation by Secreting TGF-beta3 and TSP-1 to Elicit Macrophage M2 Polarization. Cell Physiol Biochem 2018;51:2290-308.

13. Zhang K, Kaufman RJ. From endoplasmic-reticulum stress to the inflammatory response. Nature 2008;454:455-62.

14. Kim HJ, Jeong JS, Kim SR, et al. Inhibition of endoplasmic reticulum stress alleviates lipopolysaccharide-induced lung inflammation through modulation of NF-kappaB/HIF1alpha signaling pathway. Sci Rep 2013;3:1142.

15. Kim SR, Kim HJ, Kim DI, et al. Blockade of Interplay between IL-17A and Endoplasmic Reticulum Stress Attenuates LPS-Induced Lung Injury. Theranostics 2015;5:1343-62.

16. Zeng $M$, Sang W, Chen S, et al. 4-PBA inhibits LPS-induced inflammation through regulating ER stress and autophagy in acute lung injury models. Toxicol Lett 2017;271:26-37.

17. Zhang X, Goncalves R, Mosser DM. The isolation and characterization of murine macrophages. Curr Protoc Immunol 2008; Chapter 14:Unit 14.1.

18. Cross BC, Bond PJ, Sadowski PG, et al. The molecular basis for selective inhibition of unconventional mRNA splicing by an IRE1-binding small molecule. Proc Natl Acad Sci U S A 2012;109:E869-78.

19. Su X, Wang L, Song Y, et al. Inhibition of inflammatory responses by ambroxol, a mucolytic agent, in a murine model of acute lung injury induced by lipopolysaccharide. Intensive Care Med 2004;30:133-40.

20. Taki T, Masumoto H, Funamoto M, et al. Fetal mesenchymal stem cells ameliorate acute lung injury in a rat cardiopulmonary bypass model. J Thorac Cardiovasc Surg 2017;153:726-34.

21. Hussell T, Bell TJ. Alveolar macrophages: plasticity in a tissue-specific context. Nat Rev Immunol 2014;14:81-93.
22. Aggarwal NR, King LS, D'Alessio FR. Diverse macrophage populations mediate acute lung inflammation and resolution. Am J Physiol Lung Cell Mol Physiol 2014;306:L709-25.

23. Kim SH, Kwon D, Lee S, et al. Polyhexamethyleneguanidine Phosphate-Induced Cytotoxicity in Liver Cells Is Alleviated by Tauroursodeoxycholic Acid (TUDCA) via a Reduction in Endoplasmic Reticulum Stress. Cells 2019. doi: 10.3390/ cells8091023.

24. Mohan S, R PRM, Brown L, et al. Endoplasmic reticulum stress: A master regulator of metabolic syndrome. Eur J Pharmacol 2019;860:172553.

25. Chadwick SR, Lajoie P. Endoplasmic Reticulum Stress Coping Mechanisms and Lifespan Regulation in Health and Diseases. Front Cell Dev Biol 2019;7:84.

26. Aggarwal S, Ahmad I, Lam A, et al. Heme scavenging reduces pulmonary endoplasmic reticulum stress, fibrosis, and emphysema. JCI Insight 2018. doi: 10.1172/jci.insight.120694.

27. Dickens JA, Malzer E, Chambers JE, et al. Pulmonary endoplasmic reticulum stress-scars, smoke, and suffocation. FEBS J 2019;286:322-41.

28. Leonard A, Grose V, Paton AW, et al. Selective Inactivation of Intracellular BiP/GRP78 Attenuates Endothelial Inflammation and Permeability in Acute Lung Injury. Sci Rep 2019;9:2096.

29. Huang X, Xiu H, Zhang S, et al. The Role of Macrophages in the Pathogenesis of ALI/ARDS. Mediators Inflamm 2018;2018:1264913.

30. Xue X, Piao JH, Nakajima A, et al. Tumor necrosis factor alpha (TNFalpha) induces the unfolded protein response (UPR) in a reactive oxygen species (ROS)-dependent fashion, and the UPR counteracts ROS accumulation by TNFalpha. J Biol Chem 2005;280:33917-25.

31. O'Neill CM, Lu C, Corbin KL, et al. Circulating levels of IL-1B+IL-6 cause ER stress and dysfunction in islets from prediabetic male mice. Endocrinology 2013;154:3077-88.

32. Hasnain SZ, Lourie R, Das I, et al. The interplay between endoplasmic reticulum stress and inflammation. Immunol Cell Biol 2012;90:260-70.

Cite this article as: Zhao Y, Jiang Y, Chen L, Zheng X, Zhu J, Song X, Shi J, Li Y, He W. Inhibition of the endoplasmic reticulum (ER) stress-associated IRE-1/XBP-1 pathway alleviates acute lung injury via modulation of macrophage activation. J Thorac Dis 2020;12(3):284-295. doi: 10.21037/ jtd.2020.01.45 